\title{
Waveform Multiplexing using Chirp Rate Diversity for Chirp-Sequence based MIMO Radar Systems
}

Fabian Roos,

Nils Appenrodt, Jürgen Dickmann, and Christian Waldschmidt

(C) 2018 IEEE. Personal use of this material is permitted. Permission from IEEE must be obtained for all other uses, in any current or future media, including reprinting/republishing this material for advertising or promotional purposes, creating new collective works, for resale or redistribution to servers or lists, or reuse of any copyrighted component of this work in other works.

DOI: 10.1109/RWS.2018.8304946 


\title{
Waveform Multiplexing using Chirp Rate Diversity for Chirp-Sequence based MIMO Radar Systems
}

\author{
Fabian Roos", Nils Appenrodt", Jürgen Dickmann*, and Christian Waldschmidt ${ }^{\#}$ \\ ${ }^{\#}$ Institute of Microwave Engineering, Ulm University, 89081 Ulm, Germany \\ "Daimler AG, Group Research and Advanced Engineering, 89081 Ulm, Germany
}

\begin{abstract}
MIMO radar systems create a large virtual aperture to enhance the angular resolution. As a multiplexing scheme often the time-division multiplexing (TDM) procedure is chosen. The drawbacks are a reduced maximal unambiguously detectable Doppler frequency and the need to correct a phase error in angle estimation for relative radial velocities. To overcome these disadvantages a multiplexing scheme is proposed which uses different chirp rates. Every transmitting antenna is active at the same time but can be distinguished due to the different slopes of the frequency ramps.
\end{abstract}

Index Terms - MIMO radar, automotive, chirp modulation, multiplexing

\section{INTRODUCTION}

In radar systems the achievable angular resolution is directly linked to the aperture size of the antenna [1]. A common procedure to increase the aperture is to operate at a multiple-input multiple-output (MIMO) mode which results in a larger virtual aperture. Hence, an orthogonal waveform is required to assign the different signals to the corresponding antennas. Different waveforms are investigated and compared in [2] and [3].

The most commonly used multiplexing scheme is the time-division multiplexing (TDM) approach. Every transmitting antenna gets a time interval assigned at which it is active solely. The major drawback is that the time between one specific transmit antenna is active is increased by the number of different transmit antennas. This repetition time has an influence on the maximal detectable Doppler frequency [4] which is decreased by the number of transmitting elements. Additionally, if a relative radial velocity of targets is present, a motion compensation is required to compensate phase errors [5].

Using frequency-division the transmitting antennas are separated in the frequency domain such that adjacent frequency ramps do not interfere due to the anti-aliasing filter. The drawback is the larger occupied frequency range [2].

A third possibility is to use different slopes of the frequency ramps called chirp rate division multiplexing. One possible realisation is shown in [2], where each antenna transmits different ramp slopes, but mixes the received signals with the same reference signal. This leads to an increase of the noise floor due to unfocused responses spread over the beat signal.

In contrast to a fixed reference signal, the proposed multiplexing scheme mixes the received signals with the transmitted ones as in the chirp-sequence signal processing. Using this approach the distortion from other antennas can be minimised, the maximal detectable Doppler frequency is not reduced, and no phase correction is required.

\section{Chirp Rate Multiplexing}

Every transmit antenna is active at the same time, but transmits frequency ramps with different chirp rates as depicted in Fig. 1. Without loss of generality, in the following, three transmit antennas are assumed. The bandwidth $B$ is identical for each ramp to ensure the same radial resolution, only the chirp duration $T_{\mathrm{c}, k}$ with $k \in$ $[1,2,3]$ varies. As derived in [4] the phase of a transmitted frequency ramp can be described as

$$
\varphi_{\mathrm{T}}(t)=2 \pi\left[f_{0} t+\frac{1}{2} \frac{B}{T_{\mathrm{c}, k}} t^{2}-\varphi_{0}\right],
$$

with the starting frequency $f_{0}$ and an arbitrary phase offset $\varphi_{0}$. Assuming transceivers, each antenna receives the responses from all antennas. The ramps associated with the respective antennas lead to target peaks with respect to the chirp rate at different frequencies. This means that a single target is detected with the quantity of the number of transmit antennas. Those peaks will later be used to calculate the angle to the target. Unfortunately, the ramps also interfere with each other at the receiver, e.g. the ramp from antenna 1 is mixed with the ramp from antenna 2

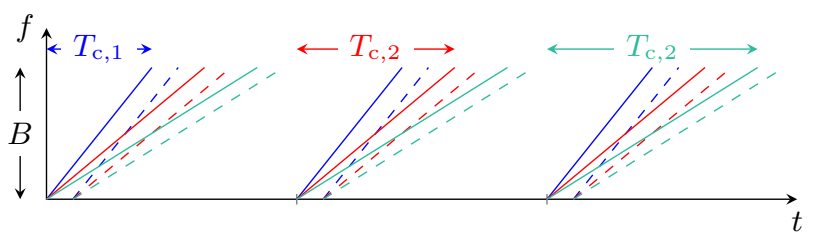

Fig. 1. Each transmit antenna is active at the same time, but transmits frequency ramps with different slopes defined by the chirp duration $T_{\mathrm{c}, k}$. 


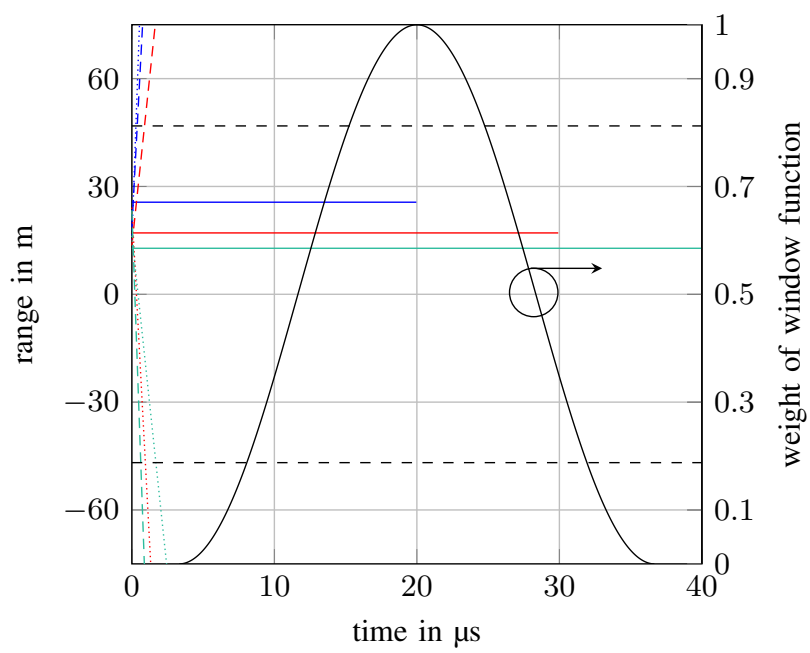

Fig. 2. The intermediate frequencies, scaled to the target range, resulting from a single target using three transmit antennas.

resulting into an interfering signal, which can be described with the phase

$$
\begin{aligned}
& \Delta \varphi_{12}(t)=\varphi_{\mathrm{T}_{1}}(t)-\varphi_{\mathrm{T}_{2}}(t-\tau) \\
& \approx 2 \pi\left\{f_{0} \tau+\frac{B}{T_{\mathrm{c}, 2}} t \tau+\frac{1}{2} \frac{B\left(T_{\mathrm{c}, 2}-T_{\mathrm{c}, 1}\right)}{T_{\mathrm{c}, 1} T_{\mathrm{c}, 2}} t^{2}\right\},
\end{aligned}
$$

assuming the simplifications from [4]. For a target with velocity $v$ and distance $R$ the time difference $\tau=2(R+v t) / \mathrm{c}$ is used as a model for a linear motion resulting in

$$
\begin{aligned}
\Delta \varphi_{12}(t)= & 2 \pi\left\{\frac{2 f_{0} R}{\mathrm{c}}+\left(\frac{2 f_{0} v}{\mathrm{c}}+\frac{2 B R}{\mathrm{c} T_{\mathrm{c}, 2}}\right) t\right. \\
& \left.+\left(\frac{2 B v}{\mathrm{c} T_{\mathrm{c}, 2}}+\frac{1}{2} \frac{B\left(T_{\mathrm{c}, 2}-T_{\mathrm{c}, 1}\right)}{T_{\mathrm{c}, 1} T_{\mathrm{c}, 2}}\right) t^{2}\right\} .
\end{aligned}
$$

The resulting frequency is not constant, but is rather linear changing. In Fig. 2 the intermediate frequencies are plotted with a $y$ axis scaled to the target range. The steepest ramp (-) is the shortest one and results in the highest frequency. The single point target is thus represented by three intermediate frequencies. The corresponding interference frequencies belonging to the first ramp slope $(--,, \ldots)$ are increasing quite fast and are after a few micro seconds outside of the filter bandwidth (- - -). If the sampled signal is weighted with a window function (-), the part affected from the interference has only a minor influence.

As each transmit antenna is active at the same time, the longest ramp determines the total ramp repetition time $T_{\mathrm{r}}$ which is directly linked to the unambiguous velocity. If the different ramp durations $T_{\mathrm{c}, k}$ are chosen in a close range,

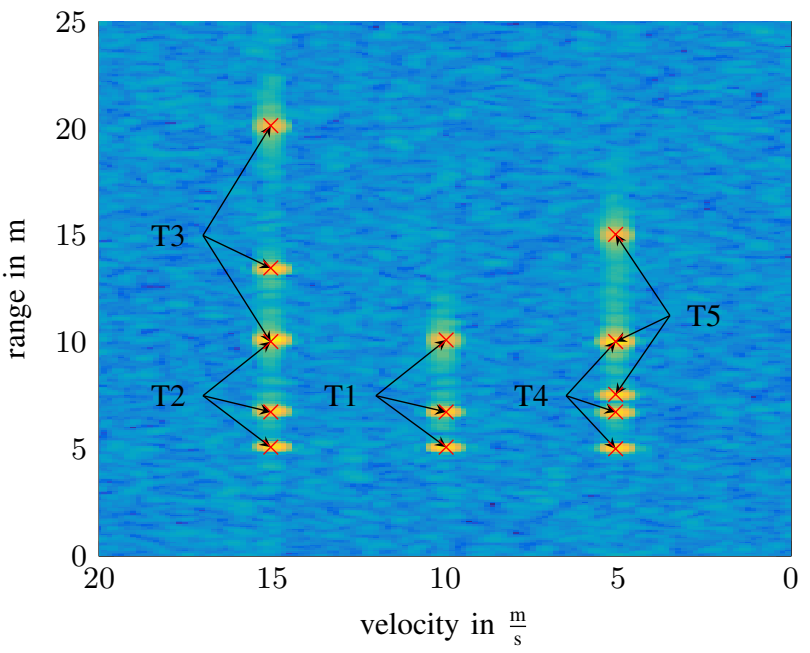

Fig. 3. Simulation of five different targets with radar parameters given in Tab. I and target parameters given in Tab. II.

$T_{\mathrm{r}}$ can be chosen considerably smaller than in the TDM case leading to a larger unambiguous velocity.

\section{Signal Processing Chain}

The transmitted signals of each antenna are combined and mixed with the received signal of each antenna. A two-dimensional Hann window function is applied to the time-domain data obtained from each antenna. For range direction the window is scaled to the longest frequency ramp. Subsequently, a two-dimensional Fourier transform is applied resulting in the range-Doppler spectrum. Zero-padding eases the later explained matching of the corresponding targets. An ordered statistic-constant false alarm rate $(O S-C F A R)$ algorithm [6] and a peak search is used to extract point targets.

Since every point target is detected $k$ times, where $k$ is the number of transmit antennas, a matching algorithm searches for targets that belong together. The velocity of the targets is always the same, but the extracted ranges depend on the ratio of the chirp durations $T_{\mathrm{c}, k}$, which is known in advance. For every detected peak it is checked if other peaks are present in the expected positions, considering a tolerance in range and velocity.

After the matching it is known which peaks belong to which target. The phase value of a peak is dependant on the respective antenna, the angle under which the target is detected, and the distance to it. With this phase difference the angle under which the target is detected can be calculated using this relation or with a conventional Bartlett beamformer. As the ramps are transmitted at the same time there is no need for a phase error correction as for TDM [5]. 


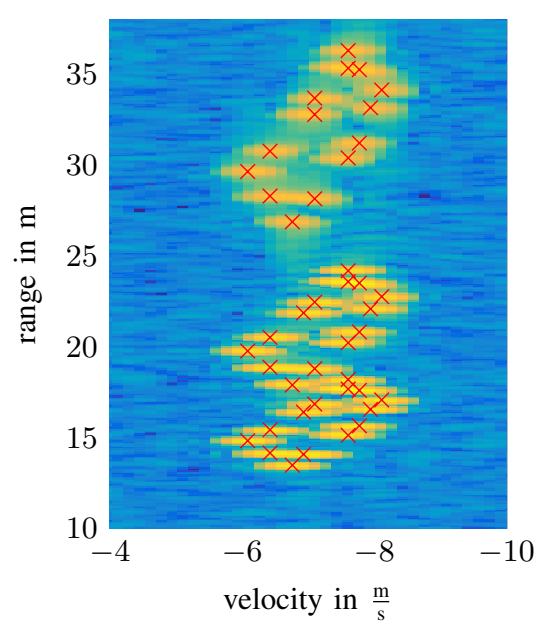

(a) Range-Doppler spectrum showing the vehicle three times. Peaks $(X)$ are detected after application of CFAR with peak search.

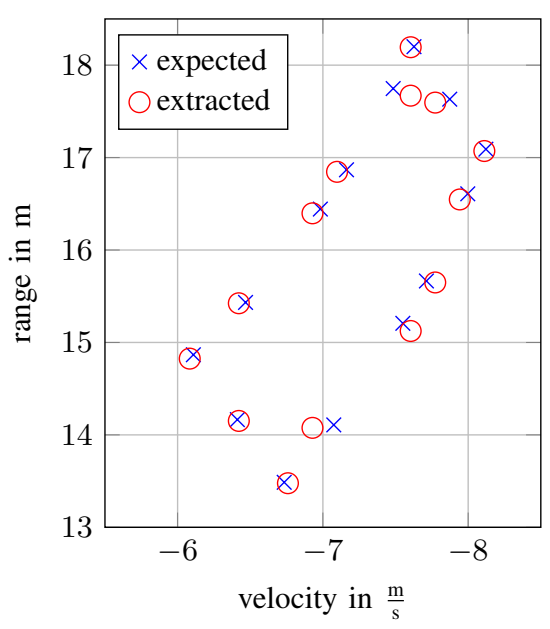

(b) The application of the matching results in the extracted $(\bigcirc)$ ranges and velocities which are compared to the expected $(\times)$ ones.

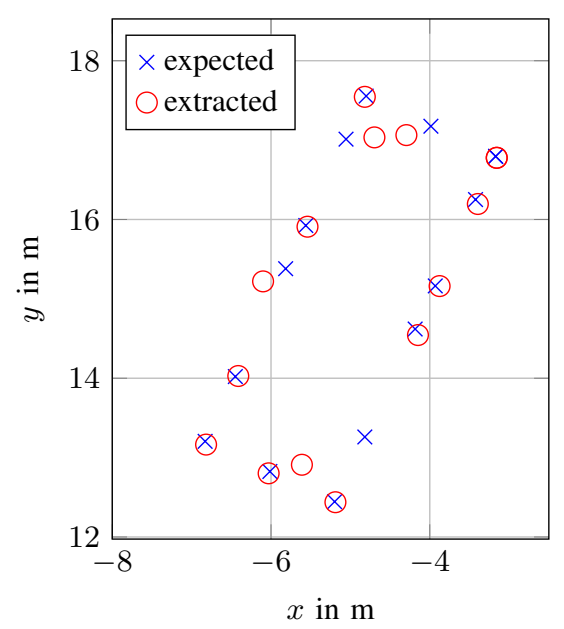

(c) Angle estimation for each extracted scattering centre shown in the $x-y$ representation.

Fig. 4. Simulation of a vehicle as in [7] to show the working matching and angle estimation for a challenging extended target.

\section{Simulation Results}

This multiplexing scheme is simulated with the parameters listed in Tab. I using three transmit antennas as assumed in Fig. 1. Additionally, additive white Gaussian noise is applied to the simulated time-domain data.

First of all, several point targets are simulated with parameters as given in Tab. II. The index 'sim' stands for the simulated and therefore expected parameter. The range-Doppler spectrum of the first antenna is shown in Fig. 3. As already mentioned in the previous section, the single target $\mathrm{T} 1$ is detected three times and the matching algorithm must assign the three peaks to one single target. The extracted target parameters are also listed in Tab. II with the index 'extr'.

The targets T2 and T3 have different ranges, but with the multiplexing scheme one peak is identical, which must be recognised by the matching algorithm. Also one peak is identical considering $\mathrm{T} 4$ and $\mathrm{T} 5$, while the angle estimation

TABLE I

SPECIFICATIONS OF CHIRP RATE MUlTIPLEXING RADAR SimUlations

\begin{tabular}{lr} 
Parameter & Value \\
\hline starting frequency $f_{0}$ & $76.5 \mathrm{GHz}$ \\
bandwidth $B$ & $1 \mathrm{GHz}$ \\
chirp duration $T_{\mathrm{c}, k}$ & $20 \mu \mathrm{s}, 30 \mu \mathrm{s}, 40 \mu \mathrm{s}$ \\
chirp repetition time $T_{\mathrm{r}}$ & $45 \mu \mathrm{s}$ \\
sampling frequency $f_{\mathrm{s}}$ & $25 \mathrm{MHz}$ \\
number of chirps $L$ & 128 \\
number of antennas & 3 \\
element distance in $\lambda$ & 0.545 \\
window function & Hann window \\
zero padding & signal length doubled
\end{tabular}

is still possible.

To consider an extended target, a vehicle is simulated using the proposed model from [7] with 14 closely positioned scattering centres modelled as point targets. In the range-Doppler spectrum in Fig. 4 (4a) the target vehicle is detected three times. The ramps with the lowest slope lead to the lowest intermediate frequencies and therefore to the targets with the lowest radial range. Also those ramp durations are higher and therefore, more samples are present which results after the Fourier transform into higher peaks noticeable in the peak colour. After matching the extracted ranges $(\bigcirc)$ and velocities are compared with the expected ones $(\times)$ in $(4 b)$. Small deviations are present due to the noise consideration and overlapping peaks, thus the peak search does not extract the ideal positions. Additionally to an effected peak position, the phase of these peaks is also influenced which is crucial for the angle estimation with, e.g., a Bartlett beamformer. This can be seen for the two closely positioned scattering centres at the nearest and farthest edge of the vehicle. For each extracted peak the angle estimation is shown in (4c).

TABLE II

EXPECTED (SIM) AND EXTRACTED (EXTR) TARGET PARAMETERS

\begin{tabular}{crrrrrr} 
Target & $r_{\text {sim }}$ & $v_{\text {sim }}$ & $\varphi_{\text {sim }}$ & $r_{\text {extr }}$ & $v_{\text {extr }}$ & $\varphi_{\text {extr }}$ \\
\hline 1 & $5 \mathrm{~m}$ & $10 \frac{\mathrm{m}}{\mathrm{s}}$ & $5^{\circ}$ & $5.09 \mathrm{~m}$ & $9.97 \frac{\mathrm{m}}{\mathrm{s}}$ & $4.93^{\circ}$ \\
2 & $5 \mathrm{~m}$ & $15 \frac{\mathrm{m}}{\mathrm{s}}$ & $12^{\circ}$ & $5.09 \mathrm{~m}$ & $15.04 \frac{\mathrm{m}}{\mathrm{s}}$ & $12.17^{\circ}$ \\
3 & $10 \mathrm{~m}$ & $15 \frac{\mathrm{m}}{\mathrm{s}}$ & $-20^{\circ}$ & $10.03 \mathrm{~m}$ & $15.04 \frac{\mathrm{m}}{\mathrm{s}}$ & $-20.12^{\circ}$ \\
4 & $5 \mathrm{~m}$ & $5 \frac{\mathrm{m}}{\mathrm{s}}$ & $10^{\circ}$ & $5.02 \mathrm{~m}$ & $5.07 \frac{\mathrm{m}}{\mathrm{s}}$ & $9.95^{\circ}$ \\
5 & $7.5 \mathrm{~m}$ & $5 \frac{\mathrm{m}}{\mathrm{s}}$ & $-10^{\circ}$ & $7.56 \mathrm{~m}$ & $5.07 \frac{\mathrm{m}}{\mathrm{s}}$ & $-10.48^{\circ}$
\end{tabular}




\section{CONCLUSION}

To ensure a high maximal detectable Doppler frequency a multiplexing scheme using different chirp rates has been presented. As the ramps are transmitted simultaneously, the phase error known from TDM MIMO does not exist. Due to the fact that every transmit antenna is active at the same time, a single target ist detected several times. A simple matching procedure is used to find peaks belonging to each other enabling an angle estimation. Using a simulation the capability to detect an extended target has been shown.

\section{REFERENCES}

[1] J. Hasch, E. Topak, R. Schnabel, T. Zwick, R. Weigel, and C. Waldschmidt, "Millimeter-Wave Technology for Automotive Radar Sensors in the $77 \mathrm{GHz}$ Frequency Band," IEEE Trans. Microwave Theory Tech., vol. 60, no. 3, pp. 845-860, Mar. 2012.

[2] J. J. M. de Wit, W. L. van Rossum, and A. J. de Jong, "Orthogonal Waveforms for FMCW MIMO Radar," in IEEE RadarCon (RADAR), May 2011, pp. 686-691.

[3] H. Sun, F. Brigui, and M. Lesturgie, "Analysis and Comparison of MIMO Radar Waveforms," in International Radar Conference, Oct. 2014, pp. 1-6.

[4] V. Winkler, "Range Doppler Detection for automotive FMCW Radars," in European Radar Conference, Oct. 2007, pp. 166-169.

[5] C. M. Schmid, R. Feger, C. Pfeffer, and A. Stelzer, "Motion Compensation and Efficient Array Design for TDMA FMCW MIMO Radar Systems," in 6th Europ. Conf. on Antennas and Propagation, Mar. 2012, pp. 1746-1750.

[6] H. Rohling, "Radar CFAR Thresholding in Clutter and Multiple Target Situations," IEEE Trans. Aerosp. Electron. Syst., vol. AES-19, no. 4, pp. 608-621, Jul. 1983.

[7] M. Bühren and B. Yang, "Automotive Radar Target List Simulation based on Reflection Center Representation of Objects," in Workshop on Intelligent Transportation (WIT), Germany, Mar. 2006. 